\title{
Qualidade da dieta de gestantes em uma unidade básica de saúde em Belém do Pará: um estudo piloto
}

\author{
Diet quality of pregnant women in a basic health unit in Belém, Pará: a pilot study \\ Késia Carolinne Ferreira Gomes ${ }^{a}$, Vivian Rodrigues Ferreira ${ }^{b}$, Daniela Lopes Gomesc \\ a Nutricionista. Universidade da Amazônia. \\ b Nutricionista. Mestre em Ciência da Saúde. Doutoranda da Universidade Federal de Ciências da Saúde de Porto Alegre (UFCSPA). \\ c Nutricionista. Especialista em Nutrição Clínica. Especialista em Obesidade e Emagrecimento. Doutoranda em Nutrição Humana pela Universidade de Brasília. \\ Docente do Curso de Nutrição do Centro Universitário de Brasília (UniCEUB).
}

RESUMO

Objetivo: Avaliar a qualidade da dieta de gestantes cadastradas em uma unidade básica de saúde em Belém do Pará. Materiais e Métodos: Estudo piloto de delineamento transversal, realizado com gestantes em uma unidade básica de saúde em Belém do Pará no ano de 2011. Foram aplicados questionário estruturado e inquérito alimentar recordatório de 24 horas em duplicata. O peso e a altura foram aferidos durante a entrevista para análise do Índice de Massa corporal (IMC). Para a avaliação da qualidade da dieta, foram analisados 10 de seus componentes de acordo com o índice da alimentação saudável (IAS).

Resultados: Foram avaliadas 25 gestantes, com média de idade de 24,2 $\pm 7,2$ anos, peso 58,6 $\pm 6,9 \mathrm{~kg}$ e IMC $25,7 \pm 3,9$. Notou-se que 32\% (8) apresentam excesso de peso. Pelo IAS, observou-se que 100\% (25) das gestantes avaliadas não possuem uma dieta adequada, sendo que 60\% (15) possuem uma dieta que necessita de modificações e 40\% (10) apresentam uma dieta inadequada.

Conclusão: A qualidade da alimentação de gestantes cadastradas em uma unidade básica de saúde em Belém do Pará se mostrou aquém das recomendações preconizadas, demostrando que $100 \%$ das gestantes possuem uma dieta inadequada.

Palavra-chave: dieta; gestantes; consumo alimentar; índice de alimentação saudável.

Objective: To evaluate the diet quality of pregnant women registered in a basic health unit in Belém, Pará.

Materials and Methods: In 2011 a cross-sectional pilot study with pregnant women was conducted in a basic health unit in Belém, Pará. A structured questionnaire and a 24-hour dietary recall in duplicate were applied. Weight and height were measured during the interview for analysis of body mass index (BMI). For the evaluation of diet quality 10 dietary components were analysed in accordance with healthy eating index (HEI).

Results: $A$ total of 25 pregnant women, with a mean age of $24.2 \pm 7.2$ years, weighing $58.6 \pm 6.9 \mathrm{~kg}$ and with a BMI of $25.7 \pm 3.9$, were evaluated. It was noted that $32 \%$ (8) were overweight. According to HEl it was observed that $100 \%$ (25) of the evaluated pregnant women did not have a proper diet, with $60 \%$ (15) having a diet that requires modifications and $40 \%$ (10) having an inadequate diet. Conclusion: The quality of nutrition of pregnant women, registered in a basic health unit in Belém, Pará, proved to be below recommendations, showing that $100 \%$ of pregnant women had an inadequate diet.

Keywords: diet; pregnant women; food consumption; healthy eating index. 


\section{INTRODUÇÃO}

A alimentação adequada é de suma importância na gestação, onde a demanda de nutrientes e energia tendem a aumentar devido ao estado de grandes transformações orgânicas ${ }^{1}$. Segundo Lucyk e Furomoto ${ }^{2}$, desequilíbrios na alimentação de gestantes podem comprometer o desenvolvimento e crescimento do feto.

O consumo inadequado de macro e micronutrientes está associado a complicações no período gestacional. No Brasil, observa-se elevada proporção de mulheres em idade reprodutiva que realizam dietas com quantidades inadequadas de nutrientes ${ }^{3}$. Estudos nacionais indicam que gestantes não apresentam valores energéticos recomendados e estado nutricional gestacional adequado, seja por déficit ou por excesso $\mathrm{O}^{4,5}$.

O índice de alimentação saudável (IAS) é uma ferramenta destinada à avaliação da qualidade da alimentação ${ }^{6}$ que apresenta dez componentes: os primeiros cinco se referem aos grupos alimentares do Guia Alimentar Americano (grãos, vegetais, frutas, leite e carnes) e os outros cinco componentes dizem respeito à gordura total, gordura saturada, colesterol, sódio e variedade da dieta. O IAS foi considerado uma ferramenta eficiente para avaliar e monitorar a qualidade global da alimentação da população.

São poucos os estudos nacionais sobre o padrão alimentar de gestantes. Em estudo realizado por Melere et al. ${ }^{7}$, foi utilizada uma versão modificada do IAS para avaliar a alimentação das gestantes, analisando também dez componentes (vegetais, feijão e outros vegetais ricos em proteínas, frutas, razão carne branca/carne vermelha, fibra, gordura trans razão poli-insaturada/saturada, cálcio, folato e ferro). O estudo de Maeba ${ }^{8}$ aplicou o IAS adaptado para gestantes e analisou dez componentes, sendo eles: grupos de alimentos da pirâmide alimentar, gordura total e saturada, colesterol, cálcio e variedade da dieta, mostrando-se o índice uma boa ferramenta para a análise global da dieta das gestantes. Além disso, apontam que a maioria das gestantes necessitava de modificações na dieta. Neste sentido, o presente estudo tem como objetivo avaliar a qualidade da dieta de gestantes cadastradas em uma unidade básica de saúde (UBS) em Belém do Pará.

\section{MATERIAIS E MÉTODOS}

Trata-se de um estudo piloto de delineamento transversal realizado em 2011 no período de julho a setembro com gestantes cadastradas em uma UBS no município de Ananindeua no Pará. O projeto de pesquisa foi aprovado pelo Comitê de Ética em Pesquisa da Universidade da Amazônia sob o protocolo no 412734/11.
A amostra foi delimitada pela demanda espontânea de gestantes sendo mantida a rotina da UBS, ou seja, o atendimento das gestantes era realizado três vezes na semana (terças, quartas e quintas). No período da efetivação da coleta de dados, a responsável pelo atendimento das gestantes se ausentou durante alguns dias, o que ocasionou um número menor do que o preconizado anteriormente (60 gestantes).

Foram convidadas a participar do estudo gestantes a partir da 4 a até a última semana de gestação. Aquelas que aceitaram participar do estudo foram orientadas a assinar o termo de consentimento livre e esclarecido referente à pesquisa. Para a avaliação socioeconômica, foi aplicado o questionário da Associação Brasileira de Empresas de Pesquisa ${ }^{9}$, que inclui perguntas sobre o perfil sócio demográfico. Para avaliar o consumo alimentar, foi aplicado o inquérito alimentar recordatório 24 horas em duplicada, sempre em dias da semana.

A altura e o peso das gestantes foram aferidos no momento da entrevista na unidade de saúde por meio de uma balança mecânica devidamente calibrada com estadiômetro acoplado. O estado nutricional foi avaliado pelo índice de massa corporal (IMC) de acordo com a metodologia de Atalah et al. ${ }^{10}$ adotadas pelo Ministério da Saúde ${ }^{11}$. Posteriormente, os dados foram interpretados pela curva de ganho de peso de gestantes e classificou-se o estado nutricional em baixo peso (IMC $<19,8 \mathrm{~kg} / \mathrm{m}^{2}$ ), eutrofia (IMC 19,8 a $\left.26 \mathrm{~kg} / \mathrm{m}^{2}\right)$, sobrepeso (IMC 26,1 a $29 \mathrm{~kg} / \mathrm{m}^{2}$ ) e obesidade (IMC $>29 \mathrm{~kg} / \mathrm{m}^{2}$ ).

As dietas foram calculadas pelo programa Dietpro versão 5.1. A média da composição nutricional dos dois recordatórios 24 horas de cada gestante foi classificada de acordo com IAS ${ }^{6}$. No presente estudo, o componente 6, que corresponde à gordura total, foi substituído por doces e açúcares devido à alta frequência do consumo desses alimentos relacionada aos "desejos" manifestados durante a gestação ${ }^{12}$.

O estudo adaptou o IAS utilizando a Pirâmide Alimentar Brasileira ${ }^{13}$ (originando componentes de 1 a 6 ).

- Componentes de 1 a 6: foram analisados os grupos da pirâmide alimentar brasileira (1 - cereais, raízes e tubérculos; 2 - legumes e verduras; 3 - frutas; 4 - leite e derivados; 5 - carnes, ovos e leguminosas; 6 - açúcares e doces); foi pontuado com o valor máximo (10) o consumo mínimo dos grupos sugerido pelo guia e com o valor mínimo (0) quando não houve o consumo. Quando houve consumo, mas não foi atingido o mínimo sugerido pela pirâmide, as participantes receberam a pontuação proporcional entre o não consumo e o consumo mínimo recomendado. 
- Componente 7 (gordura saturada): foi atribuído o valor máximo (10) à dieta que obteve um percentual de $10 \%$ ou menos de valor energético total (VET) de gordura saturada. O valor mínimo (0) foi atribuído quando a ingestão na dieta foi de $15 \%$ ou mais do seu VET.

- Componente 8 (colesterol): a pontuação máxima (10) foi atribuída quando atingido o consumo de até 300 mg/dia e a pontuação mínima (0) equivalente à ingestão de $\geq 450 \mathrm{mg} /$ dia de colesterol.

- Componente 9 (sódio): a ingestão de sódio foi pontuada com o valor máximo (10) quando ocorreu um consumo $\leq 2.400 \mathrm{mg} /$ dia e o valor mínimo (0) pelo consumo $\geq 4.800 \mathrm{mg} /$ dia na dieta.

- Componente 10 (variedade da dieta): foi pontuado levando em consideração os diferentes tipos de alimentos, atendendo uma dieta saudável, consumida durante o dia em quantidades suficientes para contribuir com pelo menos metade de uma porção de um dos grupos de alimentos. A pontuação mínima (0) foi atribuída ao consumo igual ou inferior a três alimentos; a pontuação máxima, para o consumo igual ou superior a oito alimentos (10). Foi atribuída também a pontuação proporcional entre este consumo nestas proporções.

A qualidade da dieta foi determinada segundo a soma dos pontos de todos os dez componentes, sendo a soma dos itens avaliada como: dieta inadequada ( $<51$ pontos), dieta que necessita de modificações (51 e 80 pontos) e dieta adequada ( $>80$ pontos).

Os dados foram analisados pelo programa Statistical Package for Social Sciences (SPSS) versão 16.0. As variáveis foram descritas em percentuais e medidas de tendência central e dispersão.

\section{RESULTADOS}

Da demanda atendida compreendendo 37 gestantes, 8 não aceitaram participar da pesquisa, e 4 estavam matriculadas no PSF, mas não residiam no bairro impossibilitando assim a coleta do segundo recordatório 24 horas, sendo excluídas da amostra. Participaram da pesquisa, portanto, 25 gestantes. A média de idade foi de $24,2 \pm 7,2$ anos, sendo $32 \%$ (8) com idade menor do que 20 anos, como apresentado na Tabela 1. Em relação aos dados socioeconômicos, 56\% (14) apresentaram mais de 8 anos de estudo, 88\% (22) não residiam com o cônjuge e $84 \%$ (21) encontravam-se na classe $\mathrm{C}$ de classificação de renda.

Na Tabela 2 estão apresentados os resultados do estado nutricional de acordo com o trimestre gestacional. A média de peso foi de $58,6 \pm 6,9$ e IMC $25,7 \pm 3,9$. A prevalência do excesso de peso entre as gestantes foi de $32 \%$ (8), sendo que $40 \%$ (2) estavam com excesso de peso no primeiro trimestre, $25 \%$ (5) encontram-se com sobrepeso e apenas 5\% (1) apresentam obesidade no segundo e terceiro trimestre.

Tabela 1 - Caracterização socioeconômica das gestantes em uma Unidade de Saúde de Ananindeua conforme o trimestre gestacional.

\begin{tabular}{|c|c|c|c|}
\hline Variáveis & ${ }_{(\mathrm{n})}$ trimestre & $\begin{array}{c}2^{0} \text { e } 30 \\
\text { trimestre } \\
\text { (n) }\end{array}$ & $\begin{array}{c}\text { Total } \\
(\mathrm{n})\end{array}$ \\
\hline \multicolumn{4}{|l|}{ Idade } \\
\hline$>20$ anos & 5 & 12 & 17 \\
\hline Total & 5 & 20 & 25 \\
\hline \multicolumn{4}{|l|}{ Estado Civil } \\
\hline Solteiro & 5 & 17 & 22 \\
\hline Casado & 0 & 3 & 3 \\
\hline Total & 5 & 20 & 25 \\
\hline \multicolumn{4}{|l|}{ Ocupação } \\
\hline Com ocupação & 4 & 15 & 19 \\
\hline Sem ocupação & 1 & 4 & 6 \\
\hline Total & 5 & 20 & 25 \\
\hline \multicolumn{4}{|l|}{ Classe econômica } \\
\hline D & 2 & 0 & 2 \\
\hline C2 & 1 & 10 & 11 \\
\hline C1 & 2 & 8 & 10 \\
\hline B2 & 0 & 2 & 2 \\
\hline Total & 5 & 20 & 25 \\
\hline \multicolumn{4}{|l|}{ Escolaridade } \\
\hline$<8$ anos & 4 & 7 & 11 \\
\hline$>$ 8anos & 1 & 13 & 14 \\
\hline Total & 5 & 20 & 25 \\
\hline
\end{tabular}

Tabela 2 - Caracterização do Estado Nutricional de gestantes em uma Unidade de Saúde de Ananindeua conforme o trimestre gestacional.

\begin{tabular}{l|c|c|c|}
\hline Estado Nutricional & $\begin{array}{c}\mathbf{1 0} \text { trimestre } \\
(\mathbf{n})\end{array}$ & $\begin{array}{c}\mathbf{2 0} \text { e 3o } \\
\text { trimestre } \\
(\mathbf{n})\end{array}$ & $\begin{array}{c}\text { Total } \\
(\mathbf{n})\end{array}$ \\
\hline Baixo peso & 2 & 3 & 5 \\
Adequado & 1 & 11 & 12 \\
Sobrepeso & 2 & 5 & 7 \\
Obesa & 0 & 1 & 1 \\
Total & 5 & 20 & 25 \\
\hline
\end{tabular}

Observa-se que 100\% (25) das gestantes avaliadas não possuem uma dieta adequada segundo o IAS sendo que $60 \%$ (15) possuem uma dieta que necessita de modificações e $40 \%$ (10) apresentam uma dieta inadequada conforme apresentado na Tabela 3. 
Tabela 3 - Classificação da qualidade da alimentação de gestantes segundo o índice da alimentação saudável (IAS) conforme o trimestre gestacional.

\begin{tabular}{|c|c|c|c|}
\hline $\begin{array}{l}\text { Índice de } \\
\text { alimentação saudável }\end{array}$ & $\underset{(\mathrm{n})}{10}$ trimestre & $\begin{array}{c}20 \text { e } 30 \\
\text { trimestre } \\
(\mathrm{n})\end{array}$ & $\begin{array}{c}\text { Total } \\
(\mathbf{n})\end{array}$ \\
\hline $\begin{array}{l}\text { Dieta inadequada } \\
\quad(<51 \text { pontos })\end{array}$ & 3 & 7 & 10 \\
\hline $\begin{array}{l}\text { Dieta que necessita de } \\
\text { modificações } \\
\quad \text { ( } 51 \text { e } 80 \text { pontos) }\end{array}$ & 2 & 13 & 15 \\
\hline $\begin{array}{l}\text { Dieta saudável } \\
\quad \text { (> } 80 \text { pontos) }\end{array}$ & 0 & 0 & 0 \\
\hline Total & 5 & 20 & 25 \\
\hline
\end{tabular}

\section{DISCUSSÃO}

Este estudo avaliou a qualidade da alimentação de gestantes usuárias do serviço público de saúde. Os resultados demonstram que a alimentação da população estudada é de baixa qualidade e necessita de alterações. Neste estudo, verificou-se que $32 \%$ das gestantes apresentaram excesso de peso, este dado acompanha a tendência de outros resultados. Fazio ${ }^{14}$ avaliou 187 gestantes e detectou $42,85 \%$ de excesso de peso (19,8\% com sobrepeso e $23 \%$ obesas). Na pesquisa de Brognoli et al. ${ }^{15}$, realizada com 114 gestantes em Curitiba, foram encontrados $34 \%$ de excesso de peso (24\% com sobrepeso e $10 \%$ obesas).

Segundo Mattar et al. ${ }^{16}$, o excesso de tecido adiposo materno pode afetar o feto na fase embrionária até o parto. A taxa de malformações é maior em mulheres obesas do que naquelas com diagnóstico nutricional adequado. Segundo Silva et al. ${ }^{17}$, o nível socioeconômico e cultural é a soma de vários fatores ( nível de instrução, ocupação e renda) que interferem no padrão de vida familiar, cuidado com a saúde, tipo de moradia, assistência ao pré-natal e na alimentação.

Os resultados demonstram que a alimentação das gestantes avaliadas necessita de modificações. Nossos achados corroboram com os resultados de Melere et al. ${ }^{7}$, em que gestantes avaliadas apresentaram dieta que necessitava de modificações. De acordo com Hedrinch et al. ${ }^{5}$, quanto menor a renda, menor o poder de compra e acesso a uma alimentação variada. De forma geral, a baixa escolaridade estaria relacionada com o baixo padrão socioeconômico, sendo este um potencial risco para a mãe e o feto. Além disso, o nível de escolaridade pode interferir no grau de entendimento dos cuidados de saúde necessários durante a gestação, como alimentação adequada ${ }^{18}$.

A identificação de deficiências nutricionais e de um estado nutricional inadequado permite ao nutricionista intervir precocemente com orientações individualizadas para cada caso, evitando assim aumento da morbimortalidade perinatal e neonatal $19,20,21,22$. Outros fatores podem contribuir na qualidade da alimentação, segundo Baião e Deslandes ${ }^{23}$, gestante podem restringir a alimentação por sofrerem com alguns sintomas que causam sofrimento e perda de peso. Estima-se que $50 \%$ a $80 \%$ das gestantes sofrem de náusea e 50\% vômitos ${ }^{24}$. Nossos achados demostram que 20\% (5) encontravam-se no $1^{\circ}$ trimestre gestacional, sendo este um período característico de náuseas e vômitos que podem comprometer o consumo de alimentos.

Nessa fase há ocorrências de várias transformações hormonais e emocionais que podem desencadear picacismo (compulsão de ingestão de substâncias não nutritivas), estas alterações podem levar a diminuição do apetite devido ao volume de algumas substâncias ingeridas (gelo, terra, giz, cera, papel), comprometendo mãe e feto ${ }^{25}$. Outras alterações podem ocorrer, como azia, constipação, má digestão e placa exacerbada, que pode levar a gengivite (inflamação na gengiva) na gravidez, mais comum no segundo e terceiro trimestre gestacional. Estes sintomas podem levar a restrição alimentar comprometendo assim a alimentação adequada nesta fase ${ }^{11,26}$. Apesar de não avaliarmos, estes fatores podem estar relacionados à elevada prevalência de dieta inadequada, observada no estudo.

Este estudo apresenta algumas limitações. As características socioeconômicas da população são homogêneas e não permitem extrapolar os resultados para outras populações. Outra limitação verificada foi o pequeno número de gestantes captadas durante o estudo. Apesar das limitações citadas, o IAS demonstrou ser uma ferramenta eficaz para a avaliação da qualidade da alimentação da população estudada, diferentemente de avaliações de nutrientes isoladas.

Os resultados deste estudo permitem concluir que a qualidade da alimentação da população avaliada se mostrou aquém das recomendações preconizadas, ressaltando a importância de estratégias e intervenções no âmbito de saúde pública para melhoria da alimentação nesse grupo populacional. Sugerimos que novos estudos sejam conduzidos com o intuito de investigar a qualidade da alimentação nas populações usuárias de serviços públicos de saúde, considerando que este é um grupo de maior vulnerabilidade social e biológica a fim de nortear a implementação de políticas públicas para intervir em programas de assistência pré-natal que levem informações claras e práticas a respeito da importância da alimentação adequada nesta fase da vida.

\section{REFERÊNCIAS}

1. Nascimento E, Souza SB. Avaliação da dieta de gestantes com sobrepeso. Rev Nutr 2002;15(2):173-9. http://dx.doi.org/10.1590/ S1415-52732002000200006 
2. Lucyk JM, Furumoto RM. Necessidades nutricionais e consumo alimentar na gestação: uma revisão. Comun ciênc saúde 2008;19(4):353-63.

3. Lacerda EMA, Kac G, Cunha CB, Leal MC. Consumo alimentar na gestação e no pós-parto segundo cor da pele no município do Rio de Janeiro. Rev Saúde Pública 2007;41(6):985-94. http://dx.doi. org/10.1590/S0034-89102007000600014

4. Nochieri ACM, Assumpção MF, Belmonte FAL, Leung MCA. Perfil nutricional de gestantes atendidas em primeira consulta de nutrição no pré-natal de uma instituição filantrópica de São Paulo. Mundo saúde 2008;32(4):443-51.

5. Hedrich A, Novello D, Ruviaro L, Alves J, Quintiliano DA. Perfil alimentar, estado nutricional, de saúde e condições sócio-econômicas de gestantes assistidas por centros de saúde do município de Guarapuava-PR. Rev salus 2007;1(2): 139-46.

6. Kennedy ET, Ohls J, Carlson S, Fleming K. The healthy eating index: design and applications. J Am Diet Assoc1995;95(10):1103-8. http://dx.doi.org/10.1016/S0002-8223(95)00300-2

7. Melere C, Hoffmann JF, Nunes MAA, Drehmer M, Buss C, Ozcariz SGI, Soares RM, Manzolli PP, Duncan BB, Camey SA. Índice de alimentação saudável para gestantes: adaptação para uso em gestantes brasileiras. Rev Saúde Pública 2013;47(1):20-8. http:// dx.doi.org/10.1590/S0034-89102013000100004

8. Maeda AP. Padrão de consumo alimentar de gestantes atendidas em um serviço público de pré-natal: um estudo de coorte [tese]. São Paulo: Universidade de São Paulo; 2002.

9. Associação Brasileira de Empresas de Pesquisa. Classificação econômica Brasil; 2008 [capturado em 2011 fev. 2]. Disponível em: http://www.abep.org

10. Atalah SE, Castillo LC, Castro SR, Áldea PA. Propuesta de um nuevo estándar de evaluación nutricional em embarazadas. Rev méd Chile 1997;125(12):1429-36.

11. Brasil. Ministério da Saúde. Secretaria de Atenção a Saúde. Atendimento nutricional no pré-natal e puerpério. Manual técnico. Versão preliminar. Brasília (DF): Ministério da Saúde; 2000.

12. Orloff N.C, Hormes J.M. Pickles and ice cream! Food cravings in pregnancy: hypotheses, preliminary evidence, and directions for future research. Front Psychol 2014;5(1):1-15. http://dx.doi. org/10.3389/fpsyg.2014.01076

13. Philippi ST, Latterza AR, Cruz ATR, Ribeiro LC. Pirâmide alimentar adaptada: guia para escolha dos alimentos. Rev Nutr 1999;12(1): 65-80. http://dx.doi.org/10.1590/S1415-52731999000100006
14. Fazio ES. Perfil nutricional de gestantes que recebem orientação dietética: avaliação do ganho ponderal materno total, tipo de parto e resultados perinatais [dissertação]. São Paulo: Universidade de São Paulo; 2010.

15. Brognolli AF, Neme LCH, Passoni CMS. Relação da dieta de gestantes com o estado nutricional. Cadernos da Escola de Saúde 2010;3(1):1-14.

16. Mattar R, Torloni MR, Betrán AP, Merialsi M. Obesidade e gravidez. Rev Bras Ginecol Obstet 2009;31(3):107-10. http://dx.doi. org/10.1590/S0100-72032009000300001

17. Silva ET, Caetano JA, Silva ARV. Assistência pré-natal de um serviço de atendimento secundário. Rev Bras Promoc Saude 2006;19(4): 216-23. http://dx.doi.org/10.5020/18061230.2006.p216

18. Ramos HAC, Cumam RKN. Fatores de risco para prematuridade: pesquisa documental. Esc Anna Nery Rev Enferm 2009;13(2): 297-304. http://dx.doi.org/10.1590/S1414-81452009000200009

19. Valle CP, Durce P, Ferreira CAS. Consequências fetais da obesidade gestacional. Mundo saúde 2008;32(4):537-41.

20. Silva LSV, Thiapó AP, Souza GG, Sauders C, Ramalho A. Micronutrientes na gestação e lactação. Rev bras saúde matern infant 2007;7(3):237-44. http://dx.doi.org/10.1590/s151938292007000300002

21. Paula HAA, Salvador BC, Souza CC, Cota AM, Batista MA, Pires RC, Martins PC, Cotta RMM. Atenção pré-natal em Viçosa-MG: contribuições para discussão de políticas públicas de saúde. Rev Med Minas Gerais 2008;18(3):167-74.

22. Busch LI, Silva ACP, Bosco SMD. Adequação do consumo alimentar das gestantes frequentadoras de um grupo de gestantes de um município do interior do Rio Grande do Sul. Cad Pedagog 2009;6(2):9-28.

23. Baião MR, Deslandes SF. Gravidez e comportamento alimentar em gestantes de uma comunidade urbana de baixa renda no Município do Rio de Janeiro, Brasil. Cad Saúde Pública 2008;24(11):2633-42. http://dx.doi.org/10.1590/S0102-311X2008001100018

24. Matthews A, Haas DM, O'Mathúna DP, Dowswell T, Doyle M. Interventions for nausea and vomiting in early pregnancy. Cochrane Database Syst Rev 2014;9(2):1-68. http://dx.doi. org/10.1002/14651858.cd007575.pub3

25. Dunker KLL, Alvarenga MS, Alves VPO. Transtornos alimentares e gestação: uma revisão. J Bras Psiquiatr 2009;58(1):60-8. http:// dx.doi.org/10.1590/S0047-20852009000100010

26. Cunha DDP. Doença periodontal na gestação [monografia Especialização em Periodontia]. Porto Alegre: Universidade Federal do Rio Grande do Sul; 2011. 